Recibido: 12-07-2016 Aceptado: 26-07-2016

Palabras clave: Neoliberalismo, educación. Keywords: Neoliberalism, education .

Alessandro Cavigl ia acaviglia@yahoo.com PUCP -UARM

\section{La forja del Neoliberalismo y la Educación en el Perú}

\section{Theforging of noliberalism and the education in Peru}

\author{
Alessandro Caviglia ${ }^{1}$
}

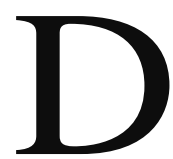
esde hace unos años se ha instaurado un sentido común en el Perú respecto de la educación, que resulta más que preocupante. Se está asumiendo, sin más, que: a) la educación pliblica debe desaparecer b) que se deben efüninar los cursos de artes y humanidades de la currícula y c) que debe de incorporarse el curso de emprendedurismo en la currícula escolar. Muchos de estos cambios se han propuesto para todos los niveles de la educación, tan to primaria, secundaria, terciaria (o técnica) y universitaria.

a) La idea de que la educación pública tiene que desaparecer ha sido señalada con toda claridad por el abogado Alfredo Bullard . Como es sabido,Bullard es uno de los maxirnos exponentes de la escuela conocida como Análisis Económico del Derecho. El .Análisis Económico del Derecho es una corriente sobre el derecho que fue fundada por Richard Posner (Posoer, 2011) en la Universidad de Ch.icago y que se presentó primero como una propuesta para el derecho privado, especialmen te el corporativo y el civil. La idea central es que los daños y reparaciones civiles se pueden cuantificar en dinero y la justicia puede ser trocada en u na transacción monetaria. Pero los seguidores de Posner han buscado trasladar esas ideas a otras áreas del derecho, especialn1ente al derecho público y postulan que cuestiones que son un derecho, como la educación, la salud yla seguridad, se pueden introducir en el mercado sin mayor problema. Lo que hacen estos teólicos es reemplazar el término "derecho" por el término "necesidad", realizando un desplazamiento del significado sustancial. Mientras que la educación, la salud y la seguridad son entenclidas como derecho, se encuentran atadas a la idea moral de dignidad humana y se presentan como una exigencia del Estado elgarantizarlos. En cambio, si se presentan como "necesidades" se puede señalar que se trata de procesos que pueden ser cubiertos por el mercado,con lo cual el Estado no tiene que garantizar su satisfacción. Con ello se consigue algo adicional, que es el descargar al Estado en su máxima expresión, de manera que éste ya no tenga a a su cargo ni la educación, ni la salud ni la seguridad. 
El argumento de Bullard en contta de la educación pública consjste en que la razón por la cual el Perú se encuentra en uno de Los últimos lugares en educación en la región se debe a la deñciencia de la educación pública. La propuesta entonces es fa siguiente: eliminar las escuelas públicas, priYatizán dolas en su totalidad y darles bc> nos a los padres de familia para que ellos elijan en cuál escuela colocar a. sus hijos. Dichos bonos se atari.an a un crédito de carácter bancario, de manera que los padres de familia y sus hijos c::omraedan una deuda banca ria ${ }^{1}$.

b) La idea de la eliminación de las arres y las humarudades de la currícula de las escuelas y universidades resul ra ser una medida en auge, pero sumamente preoaipanre $r$ peligrosa. En las escuelas se ha estado asumiendo una política de intcnsificació $\mathrm{n}$ de los cursos razooamiento matemático y razonamiento verbaJ en desmedro del ju ego, de las artes y las humanidad. Algu nos teóricos de la educación, asi como algunos maestros y maescr_as enfatizan la idea de que los niilos $\Gamma$ l s niñas deben aprender a leer y escribir a muy temprana edad y ciue, en pos de ese- objeúvo

1 Esta idea seensayó en ChUc durante In dicraduru deAugusto Pinocht! y fue impleml!ntada por los llilmodos "Chicago Boys :quienes IJewron a cabo las medidas neolfberal es en Chile durante dicha díctadurn. Hace unos años, alcaldes y cstudianteS escolares $y$ universitarios tuvieron que llevnr adelanie Ulla sede de mamfestadones en fas calles contra estas medidas m la educación y él segun do gobierno de Míchelle Bachelet ha reuído que tomar medídu en dirt>c.ióo de desmantelar la medidas de privatización de las escuelas públicas. Entre las figUrab mli\$ representaliv;is del movimiento en contra de.las medidlls neolibc.rales en educacióh destac hi entonces presiden\a de la Feder.tdón de.Estud.íames de Chile (FECH), Camila Vallejo.

Estomuestra queloqueestáproponiendo Sullard no es nuevo, ha sido implementado en Chile, baJO una djétaduta, $y$ que hn frato • \$;1dototalmente; las medidas que. el JurisLll peruano presenta han frncasado ya, igualque tán frocas:tndo las AflPs en-Chile yqueen nuestro contexto tienen tanta ncogida, perp también muchos de tr.ictores, encontrándose actualmente en el ojo del debate público en el Perú.

Respecto de las afirmaciones de 8ulhird Cf bnps:I/wwwyó1111tbt • cow/wnrch!Y::HkGneHPFJJ(8 (vi.slt ijdo el 7/27/2016). Pero como no e3 un Intelectual muy original, sus ideas son una copia de las del economista Milton Ftiedman.Sobre las ideas de Friedman, $C f$. 3t1r9:Ilwww.d!!lito.org!la-privali Zí1c/O1J: d!.d_ ciQ11 \{visit11do el 7'127/2016). deben se sacriEcru: el juego y el fomento de la imagjnacióo (Nussbaarn, 2010 f.

E.1 objetivo de este pensamiento es el de generar individnos que sean piezas que acepten )' p.ongan en fo.ociooaroiento el mercado, en vez de personas y ciudadanos que puedan imaginar una sociedad diferente, donde los de(echos de todos sean respetados y la des.igualdad socioeconórnica se vaya rnduciendo.

La e.pul.s.ión de Ja Imaginación, además, conducea la erradicación de Ja mente ylos coraxones delas personas la idea y el senti.miento de solidaridad. Este ataque sistemático contra la solidaridad se reali por medio de forjar en las personas dos ideas fundamentales. En primer lugar, se genera la idea de que no vale lapena prc $<$ )CUparse por la suerte del otro. Cada cual debe velar, <le acuerdo a esca idea, por sí mismo. La creencia de Th0mas Hobbes, según la cuaJ Jas personas serían por naruraleza autointeresados (Hobbes, 2004) se ha instalado can radicalmente en los jóvenes fruto de este tipo de educación que dlos mismos señalan que hasta el gesto de abnegación de una madre que deja de comer para alimentrtr a sus hijos no representa un sencimieri.tn altruista, sino el sentimtento autoioteresado de verse como hhuen.a madre".

En.segi.lndo lugar,no sólo- seha generado u na "Vrisióa particular d.e las relaciones interpersonales dentro de la sociedadt sino que se han populariza ideas de carácter legislativo y políticas ad,rersas a la solidaridad y las políticas que las defienden. De esra manera, los jóvenes universitario s )' no universi'tarios,demás degran parte dela población, perciben como legítimo eJ. proteger Las patents médic.as y que eJ Estado no debería producir medicina genérica ea noi:nbre de la propiedad intelearua los esfuerzos de los investigadores médicos y de su esfuerzo en los estudios durante

2 Para vei: en. acción ese talante en contra delfomento de la imaginación en la escuela.. lase la noveln Tiempos difíciles de Cliarles Die kens.R. specto de In import;'Il\Cia delas I\rtesylashumanidades en la educación. 


\section{UNTFÉ - EPG}

su formación ${ }^{1}$. Además las propuestas en contra de la educación y la salud pública Ya.o ganando cada vez mas terreno a favor de fas ideas de la privatiz-ación. El que la población acepte cada vez más la reducción de la solidaridad de la vida social el desmantelamiento de las políticas que la sostienen (el gue las personas cons.ide.ren que el presupuesto asignado a la educación y a La salud debe de reducirse, asf como la permisibilidad ame la evasión de Impuestos, especialmente de parte de las grandes empresas) e:s un efecto de la implement.1.ción cultura neolibentl que seinstalado en nuestra sociedad. ${ }^{1}$

Una consecuencia nefasta de este sentido común neolibera.l adverso a la solidaridad que ha instalado en elpaís esla reacción frente a la violencia interna durnnce los 80 y 90, y el arrinconamiento político que ha sufrido el discurso de la Comisión de Ja Verdad y Reconciliación.y 1-a reacción ante el Informe I7i nal. El hecho de en las escudas no se enseñe esa dolorosa etapa dela historia reciente y d qLle en la última camptña electoral (2016) no haya aparecido en el debate político, muestra una gran carencia de solidaridad. El c1ue algunos políricos y líderes de opinión estén aimmiendo la agenda de la ureconciJíacidn sin justi <::ia" muestra claramente lo devaluada que se encuentra la solidaridad. Ese tema de la reconcíliación sin justi<;ia ha sido puesto por el fojimorismo en e.I. debate presidencial y ha estado ganando plausibilidad cada vez más amplia. Pero esto supone 1ue las vícnmas de ta violencia $\Gamma$ los deudos Cf el Perú no cuentan en absoluto. La empatfa $y$ la solidaridad se hfill desvanecido por completo. Incluso, desde Ja academia, estos sentimientos han sido expulsados, especialmente por algunos destacados oemificos políticos liderados por Martín Tanaka 5 .

3 AJ rpecto. véase los debates en tQmo a Ja fün1a del Tratado TransPácllico(TTP).

4 Relwecto de lu lmplcmenl:Ic!Ón del neoliberalismo en lbs Estados Unidos vase el documental de Noam Chomsl.'Y titulado Reqwem far the Amcricu11 Orenm. Además. del mismo Chomsky, véase Chomsky. N; IJI lumeficio ts lo qui: cue11tc1. Nt!()/iberalismoy orden 111t111difl, Barcelona:Critica, 2003.

5 Tanaka escribio haceun par de años. con motivo de la conmemo cació1i délos 10 nr1osdela éntrcga del lnfonne Final dela CVR. un e) El debate sobre la mcnrporación del curso de "ernpændedurismo" llevado a cabo en el 1 \linísceriode Educación yquerovocierra repercusión en las redes sociales $^{6}$. Ciertamente, el curso de cmprendedurismo ya se estuvo impartiendo, pero baJO el rótulo de Educación para clTu.bajo. La idea del emprendcdurismo es que l.a.s personas se vean como empren<le<loles económicos en el mercado más que como ciudadanos. Además) presupone La creencia falsn de que basta con el esfuerzo persona.1 para tener éxito en la arena económica. Ellema del emprendedurismo no es Gtro que "el esfuerzo es éxito". Y u na de las exigencias deJ mismo es que el modelo económico no varíe. Es por eJlo que una sode<lad de emprendedores es aquella en la que d pedido de la población a los político.s en una contienda electoral no puede ser otro que "Sr. Candidato, no me muevas el modelo".

Esta concepción de la educación orientada hacia el emprendimie.nto nene ,años problemas, entre los gue hay que destacar dos. El primero es que no forma ciudadanos, .mientras que el segundo (de cacicrer m::ís instturnental) es que no asegura el éxito de las personas en el mercado. Si nos enfocamos en el primero de estos ptoblemas podremos ver que el que la escuela se comprometa con el cmprendedurismo muestra una acritud decidida por formar agentes económicos pern no ciudadanos. Cuando l s personas son vistas sólo como agentes económicos, lo gue enfocamos es su destreza en el mercado. Lo gue evaluamos es si tienen o np éxiro en la contienda económica. Además, Jas esrnclisdcas )1 los informes de crecirnicnro econórnico se superponen \$Obre las poüricas, de maneta que la desigualdad es lo que menos cuenca. Se afirma que hay que tornar

arlfeulf1 que justllic; aha d arrinconamiento del dh;urso de la CVR en el droatcpolltko ac;tuaL Cj. TANAKA. Martín;Lasnmb1giieda des del IFde Ja CV.R mla explfmci.ón, en httpilllrevjstaargumeotos $\mathrm{J} 2 \mathrm{t}$ :Larticulps/las-ambiwiedades-del-if-de-la-m en·la·exuL (visita 7127/2016)

6 Debate en el que pt1rticiparo11 Susana Pr1sanco y quJtm escribe (dendicndo In iden de que d emprendedurimo no debería incluirse por.ser u11a vuelta de tuerca más 11 favor de la implementa dón del neoHberali\$mO en tl Perú)y Daniel Salas(quién defendfo al mprendedurismo) no dejó deser acalorado. 
tales y cuales medidas porque ellas fortalecen a las empresas, no porque beneficiarán a los menos aventajados de la sociedad. La idea es gue si a las empresas les va bien, a todos nos irá de maravilla. Pero esa afirmación es contrafáctica.

Formar emprendedores no es igual a forrnar ciudadanos. Se afirma que en principio un emprendedor no requiere ni siguiera terminar la secundaria. Basta con que lea un poco y sepa sumar y restar. El resto ele aspectos, como una formación humanista más amplia (como la historia, la literatura o la filosofía) es completamente prescindible. La formación de un ciudadano supone un esfuerzo humanista y artístico de mayor envergadura. Un ciudadano adecuadamente es alguien que es capaz de entender su lugar en la sociedad y en el mundo en el que vive. De esa comprensión puede detectar las injusticias y plantear las reformas necesarias, a fin de que el mercado sea ubicado dentm de límites muy precisos. Para decirlo en pocas palabras, la diferencia entre un ciudadano y un emprendedor resíde en que el ptirnero es capaz de percibir la necesidad de que los ciudadanos debat.an el lugar que debe tener el mercado y el Estado, en cambio el emprendedor no percibe la diferencia entre la sociedad y el mercado.

El segundo problema de que la escuela se dedique a formar emprendedores es de carácter instrumental. Se supone que la aspiración de un emprendedor es tener éxito en el mercado. Sin embargo, el sistema social se encuentra articulado de tal manera que las élites empresariales se encuentran aliadas al poder politico, de modo que nunca dejarán que los pobres prosperen. Las reglas del neoliberalismo se apartan en esto de las del capitalismo clásico del siglo XVIII, tal como lo presenta Adam Smith en su Riqueza de las Naciones. Mientras que el capitalismo señala que la competencia en el mercado debe ser libre y que el Estado no debe intervenir para favorecer a unos en desmedro de otros, el neoliberalismo tiene reglas diferentes.

El neoliberalismo se basa en la idea de que las élites utilizarán el poder político para acomodar el mercado a su favor. Un ejemplo de ello lo constituye la crisis norteamericana del 2008. Cuando los bancos entraron en crisis, el Estado los rescató. Bajo las reglas del capitalismo, eso no hubiese sido posible, pues el Estado no podría intervenir en la economía y debía dejar que los bancos quebrasen. Pero como lo que se impuso fue el neoliberalismo, lo que sucedió es que el Estado intervino a rescatar a las élites y hundir más a la gente, desalojándolas de sus casas.

De esta manera, el emprendedurismo forma parte de un discurso que sirve de apoyo social al oeoliberalismo. Así como, según Max IXieber, el protestantismo es el espíritu del capitalismo, podemos señalar que el emprendedurismo es el espíritu del neoliberalismo. La idea de l'Veber era que el capitalismo requería ciertas transformaciones en la conducta de las personas, como la búsqueda de la ganancia, el ahorro y el esfuerzo en el trabajo, gue no bastaba con las expectativas económicas para lograr tales cambios. Es por ello que el protestantismo sirvió de base motivacional debido a que dotaba de un discmso religioso según el cual e1 trabajo y la prosperidad resultaban ser bendición di,rjoa (Weber, 2001). De la misma manera, el emprendedutismo sirve de discurso motivacional (o deideología) para gue las personas se comprometan con el neo.liberalismo (Honneth, 2009).

Uno de los resultados del discurso del emprendedor es queeldebate politico en economía ha dado un giro sustancialmente nefasto. Hemos pasado del debate centrado en la desigualdad y en la disminución de la pobreza al debate centrado al crecimiento. De manera que la exigencia de la igualdad social ha quedado desplazado por la búsqueda del crecimien to delas empresas. Con este cambio de tema en el debate, lo gue sucede es que la exigencia de la igualdad queda completamente a la deriva de los imperativos neoliberales. Es por ello que en la práctica, la politica en el Perú pasó del combate contra la pobreza al combate contra los pobres. Esto es lo gue significó la campaña de esterilizaciones forzadas durantela dictadura de los 


\section{6 \\ UNIFÉ -EPG}

90: la idea era evitar que los pobres se reproduzcan en vez de sacarlos de la pobreza modificando el esquema económico, social y político que los mantenía en esa situación de miseria.

\section{2}

Desde hace unas décadas el término "gJ obali zación" ha adqLúrido carta de ciudadanía tanto en el lenguaje académico, como en el periodístico y en el coloquial. Este es utilizad o para referirse a un conjunto de fenómenos, como la articulación de un mercado mundial, la circulación de los flujos financieros a lo largo del globo, la comunicación de información, conocimientos $v$ tecnología a lo largo y ancho del planeta, y a la gJobalización de la justicia a través de la Corte de la Haya, el Sistema de Protección Internacional de los Derechos llumanos, la creación de una opinión pública transnacional y/o global, entre otros fenómenos. Pero, en un sentido central, por globalización nos referimos a la creación de u n mercado mu ndial donde se producen e intercambian mercancías y se producen flujos financieros cada vez de manera más acelerada.

Respecto de cuándo tuvo su inicio el fenómeno de la globalización, existe un debate que no halle! $<<\mathrm{ldO}$ a una conclusión definitiva. Una de las posiciones en el debate señala que ésta se inició con el encuentro de europeos y americanos en el cambio de siglos A.V y Arvl; mientras que una segunda posición señala que ésta se produce en el siglo XVlll al momento de producirse la "revolución industrial", la cual trajo consigo la producción de mercancías a gran escala gracias al reemplazo de las factorias por las fábricas y la incorporación de $l a$ máquina en el proceso de producción.

Finalmente, una tercera posición señala que la globalización e inicia a partir de la caída del Muro de Berlín y la expan sión del jnternet, que ha permitido generar una comunicación y un mercado globalizado y una producción de mercancías y de servicios de manera rransnacionalizada. No es nuestro objetivo decerrninar cuál de las tres posiciones respecto del inicio de la globalización tiene la razón. Pero sí podemos señalar este fenómeno que ha modificado las relaciones de poder que tienen los Estados nacionales territoriaLizados en relación a la economía.

Hoy en día se puede percibir que la posición de los Estados, respecto del poder, se ha mocü ficado por medio de Ja constitu ción de la implementaci ón del mercado global autorregulado (es decir, el tipo de mercado mundial que fusiona todos los mercados nacionales en uno, que se encuentra regulado por sí mismo - por medio de la ley de la oferta y la demanda-, y en el cual los Estados no intervienen). Con ello, las relaciones entre política y economía se han invertido perversamente. Por tal motivo, las relaciones entre las personas a lo largo del globo se encuentran condicionadas sustanrivamente de tal modo que los y las implicados puedan hacer algo al respecto.

\section{3}

Con el propósito de ofrecernos una pista de investigación respecto de las relaciones económicas gue se desarrollan en las sociedades contemporáneas, así como para entender su dinámjca y modi6caciones, Karl Polan)i (2010) escribió tanto sobre dichas relaciones como sobre el fenómeno por el cual fueron confinadas al interior de mercado.Am parado en la investigación histórica, sociológica )' antropológica que ruvo a su disposición logró explicar que la economía incluye wi conjunto de elementos que incorporan intercambios recíprocos, ideas de índole moral y relaciones mercantiles. Estas intuiciones fueron reforzadas por los estudios desarrollados por la antropología económica (Mauss, 2009 y Sahlins, 1983).

Esta complejid ad de las relaciones ccononucas tiene como objetivo comprender la dinámica de la teoría de los mercados autorregulados por medio de la idea de arraigo y 
los intentos fallidos de desarrajgo llevados a cabo por los partidarios del neoli beralismo económjco.

El concepto de "mercados autorregulados" expresalaideasegúnlacuallosmercadosseregulan a sí mismos $r$ no requieren de la intervención de ni nguna jnstancia externa, como podría ser el Estado- para funcionar adecuadamente. Dicha ídea fue expresada con toda su claridad por Adam Smith en su Riqueza <le las Naciones, señalando que los mercados no requieren de la intervención del Estado pata funcionar adecuadamente, sino que se regulan por sí mismos por una suerte de "mano invisible" que no sería otra cosa que la ley de Ja oferta y la demanda. El proyecto dd neoliberalismo económico consiste en fusionar los marcados nacionales en un mercado mundial.

El concepto de arraigo expresa la idea según la cual a) la economía se enciende como intercam bios recíprocos de bjenes, flujo de reconocimiento moral e intercambio mercantil, a la vez que se encuen tra b) empotrada en las sociedades concretas. El ascenso de las ideas neoüberales durante el siglo XIX, como producto de los cambios de la revolución industrial durante eJ siglo XVUI, produ jo transformaciones tanto en Ja forma de entender la econonúa como en la relación entre la economía y la sociedad. La rrnnsformación central consistió en el paso de una econonúa dirigida a la subsistencia a una economía dirigida a Ja gananóa. De esce cambio se derivaron una serie de Otros cambíos.

Por un lado, la economía fue despojada de su complejidad y fue reducida a la red de mercados autorregulados. De otra parte, intentaron desarraigar la economía de la sociedad, prod uciendo un proceso de desin tegración social. El camino que siguieron para tratar de llevar adelan te este objetilO fue generar mercados perfectamen te autorregulados, pero como el llevar a cabo este proyecto llevaría a la sociedad a un abismo, la misma sociedad, empezando por los mismos empresarios, no permiten la consolidación de los mercados autorregulados se concrete, y tampoco se concrete el desarraigo de la economía respecto de la sociedad, ea un proceso que Polanyj denominó "doble movimien to".

Los mismos empresarios exigen que el Estado inrervenga para poder resolver las dificultades de los mercados. Pero esta imposibilidad práctica de La consolidación de los mercados autorregulados es utilizada por los defensores del neoliberalismo como un acicate para su retórica ideológica. En cambio de señalar que de llevarse a cabo el proyecto que tienen en ciernes, se conduciría a la sociedad en su conjnllto al abjsmo y es $;:>$ or eso que la sociedad, empezando por Los empresarios, dan marcha atrás;en cambio de ser1alar eso, Jo que afuman es que Ja acción de agentes $i<$ leologizados y partidarios de la intervención del Estado, no permiten la concreción del proyecto que defienden e impiden a la sociedad de los beneficios que supuestamente prod ucirían los mercados completamente autorreguJados, desarraigados de la sociedad y dominadores de la misma.

Al mismo tiempo,losimpulsores del proyecto neoliberal, buscaron una manera de asegurar los mercados autorregulados nacionales a traYés de la forjación de un sociedad mundial de mercado.

Para ello crearon el Uamado "patrón oro", por mectio del cual todos los Estados tendrían que respaldar sus monedas en el oro, de tal manera que, entre otras cosas, podrían tener un sistema de equivalencia de precios mundial $r$ podrían regular .la existencia de circulante a través de la cantidad de reservas en oro que tuviesen los bancos centrales nacionales.

Esto permitió a la transnacio nalización de la inversión, del comercio y de los Rujos fiilancieros, de manera que ya no se encontraban sujetas a los mercados nacionales, sino que podían fluir por una sociedad de mercado mundial. Con ello, el esfuerzo fue desarraigar el mercado y consolidar la transformación de la sociedad en una sociedad de mercado. Lo característico de una sociedad de mercado es que en eUa sucede que en vez de que el mercado se empotre en un contexto social sucede lo contrario, a saber, la sociedad se encuentra 

gobernada por las relaciones de un mercado global autorregu lado.

Pero corno este proceso conduce a 10 desintegración social, las sociedades europeas de fines del siglo XIX decidieron dat marcha amis ame el abismo que veían venir. Así que los estados $<$ lecidierou elevar tos aranceles para proteger sus producciones locales y emprendieron una política oeocolonial en África, con el objetivo de incrementar su mercado interno.

Esto los condujo a una conflagraci ón mundial (la Primera Guerra Mundial) después de un sjglo XIX prácticamente caren te de eventos bélicos. Terminada la Primera Guerra> la consecución del ptoyecro neolibe:ral de consciruir un merca.do mundial autorregulado produ jo la gran depresjón del 29 y finalmente al ascenso del fascismo en la década de los 30. El fascismo representó una respuesta radical en contra del proyecto neolibe.rn.l de tal magnitud que a parir del 45 h11sta el 89 se impusieron las ideas d.e Keynes y el orden rnunrual westfwaao-keynesiano, excepto en EEUU. con Reagan e 1nglace.mi con Thatcher, donde se reactualizó el imeoco neolibera1 de rn e.rcad os autorregulados a partirde 1980.

Junto con el efecto q,ue tuvo el ascenso del fascismo y la. segunda guerra mundial, se hace necesario señalar que la Revolución Rusa de 1917 cond ujo a fortalecer la idea según la cual era necesario consolidar el llamado Estado Nacional Keyoesiano. Díc::ha revolución no sólo modificó las re'laciones económicas al interior de los países per tenecientes al bloque sovíético, sino qtie 'U impacto se dejó sentir en el mundo entero.

D.n Europa occidental $y$ en Estados Unidos hizo que las ideas de Keynes vencieran a las ideas de los defensores de los mercados autorregulados. Keples defendía la idea de que, si bien no debería desecharse los mer<:ados, era ímportame que los estados intervinieran para i ncrodncir regulaciones. Estas ideas tenían como objeto evitar que las condiciones sociales sean p.ropkias para que la prédic y la práctica de los partidos comunistas prospe rert, además de para que no se consumara la desintegración social que suponía la insrauracíón de Los mercados aucor.relados propuestos por los defensores del proyecto neoliberaF.

De esta manera, la Revolución del 17 transforma en capitalismo en esos países (Negri, 2003). En el eomnces Uamado "'tercer mundo'» la fomia en que impactó la Revolución de Ocrubre fue a través de la consolidación de gobiernos desacrollistas quti! dedclieron generar las bases materiales del bienestar social desde el Estado. La gran mayoria. de esros gobiernos fueron dictaduras, como el caso deVelasco en Perú oNasser en Egipto.

Las ideas de Kevoes no fueron el único efecto que tuvo la Revolución Rusa al interior de la economía capitalista. El otro efecto fue lo que los economistas regulacionistas francesestt denominaron 'Forclismou.

Este fenómeno despliega una idea que se enc11entra en Marx, idea según la cual, una condición necesaria para que la economía cap.italik>ta pueda seguir funcioam do es evitando la sobreproducción. I....a existencia de ella llevaria a dedive el sistema capitalista debido a que elprecio de los productos se viene aba \}o $_{\text {y }}$ haria quebrar a las emptesas.

Según este pensamiento es necesario gue eJ mismo capitalismo pueda crear un mercado CJUe pueda absorber la producdó.o pagando el rr.abajo $<$ ie los ti:abajadores de las fábricas de modo tal que ellos puedan comprar los bienes producidos por la economía capitalista.

El Fotdismo, junto con las ideas de Keynes aparecieron en las sociedades capitalistas desde el fin de la Segunda Guerra Mundial hasta la eaída

7 8n1re los defertsores de: los merados autotreg,uJados du.rllntc lnic.ios del sigio XX encontramos a \fon Misses y Von Hayok.

11 La llamada "'Teorla de la regulac::íón"surge en Francia a partir de los años 70. Una de las obras imblemáticas de La misma es el hbro de Aglietta,A "[hepryofCopitalist Reg11latio11 publicado en 1976. A rsté cconomlsta siguieran ntros 'omo Bo)'er y Llpietí.El contexto en el que esta teoría s(! origina se encuentra marcado por la cml del petréleo de losaños 70 y el consecuente cuestionamicnto dC' lai. teorías de Keynes.Esta es(u ela econórn ica desarroUó lo,s conceptos cenitales de "FordiSJ;í:lo" y "Postfordismo dasarrofl:ando la reori7.a. ción de las transformaciones poHtlco-econórnicas del capitallsmo del\$gloXX.. 
del Muro de Berlín. De tal manera ciue, ambas cos ! i jun tas, consoliJaron el llamado 'Estado de Bien estar" c¡ue subsistió en Eu ropa hasta 19.89 y en EE.UU. e lnglatena hasta 1980. El Fordismo representó lo que se u ma "la época dorada del capiLalismo" $\Gamma$ ha sido estuc:üado de manera deIJada por Michcl Foucault en sus lecciones recogidas en El nacimien to de la biopolítica_

Durante la décadn a los 70 se produjo la lhtmada "crisis del petróleo" (en 1973 y 1979), lo que llevó al declive dd Estado Keynesiano. En 1973 se produjo la llamada pri mera gran crisis dd petróleo, a raíz de la decisión de los paises árabes productores de petróleo de no l'cnder más petróleo a los paises que habían apoyado a Israel (en tre los que se contaba n Estados Unidos y los países de Eu ropa Occidental) durante la guerra que sostuvo dicho país con Siria y Egipto.

La mayoría de estos pafaes ára bes pertenecian a la OPEP (Organización de Países Eportadorcs dePetróleo) "',guesehabfa constituido en 196-0.Con esto se produjo un alza en el precio del petróleo $\mathrm{V}$ ana inflación en los paises industrializados, puesto que tenían una grao dependencia del crudo. La framacfa segunda crisic; del petróJeo se produjo encre 1979 y 981 debido a la re"\·olución iraní y la guerra enrre Irán e lrak. El 8 de sptiembre de 1978 se dio el llamado viernes negro en Tehe.rátl, en el que se desató una rerłl ta contra el Shs. lfl cuI fue repr1mida por el ejérciro. En noviembi:e 37,000 crabajadores de las refmerias se declararon eo huclf.;.l y en enero de $\mathrm{J} 979$ el Sha huye del país. El 22<le septiembre de 1980 1ráo inicia LrOa guerra con L:ak, lo cual congelal'On las exportaciones de petróleo íraní con la C()nsecuente subiJ:a del precio.

En 1980 suben al gobierno Donald Reagnn y Margarech Thatcher en EE.UU e Inglaterra respectivamente. quienes impusieron gobiernos conservadoresyreemplazaron elmodelokeynesiano por eJ neoliberal de meT-cados auronegulados,

Ln OPEP se coostltuyó el 14 deseptiembre de L960.integradn por Vt>nezuda.. Arabi<1 Saodíhl, Tran, lrok )' Kuwait.Posteriormente se lntegmaJl a la Organización Qatar fl961), Libiu (1962), 1riiratos nr;;ihcs Unidos (1967).Argelia (1969) y Nigerlo (1971). comenzaron una polidca de privatizaciones de empresas y de reJ ucción del Estado. A la caíd!\ del 1vfuro de Berlín, calló en desgrada en e1 resto de Eu ropa el modelo keyoesiano de sociedades de bienestar y de mercados parcialmente regulados por el Estado, y el neol!bcralismo echó a rodar su proyecto de mercado mundial autoa:egulndo >' de sociedad de mercado mundial, a la v-ez 9ue reemplazó el "Estado de bienestar" por el "Esrado de inseguridad" (Frnscr, 2008). A su vez. en la ex -Unión Soviética se fueron implementando lns modi6caciones en la estructura bás1ca de 111 sociedad necesarias pa ra hacer que el proyect0 neoliberal se asiente en esa región de Europa )' As1a.La Perestroika rlc $>$ s gobiernos de Gorbachov ryelsin foeron decisivos para e.so.ti'

Al mismo tiempo, en los países del antiguo "tercer mundo' fracasó el proyecto del Estado desarrollista. Deesremodo, el modelo de m('trcados aucorregulados resulta siendo la e presióo más sjgnificativa de lo que se entiende hoyen día como globalización Y> más precisamen te, globalización financiera. Pero, desde entonces, aguello que Polanyi denominó "doble rnovimienro» fue neutralizado y se ha impuesto un movimien ro qnidireccionaJ a nivel global, de manera tal que la esca1ada neolibei:al pai;ece oo rener foeo:a exretnas de contención ${ }^{11}$. Entre otras razones, esta ausencii;t de fuerzas de con te.ación se debe a la cafdn del bloque soviético. Pero la misma siruación ha hecho surgir dos fenómenos globales imporrnntes. De una parte, los Estados Unidos de América han visto debilitado la posibUidad seguir sosteniendo su hegemonía sin competencia. EJ mundo global 6.nancierizado ha hecho visto La emergencia de auevs potencias especialrnente la China.Por otra parte, La llamada t•Guerra Fría" se ha reactualizado a través de la con.figuración del eje Rusia, Irán y los paises del ALBA, enae otros.

lO La historia de la Implemeotaclon del proyecto neoUberaJ en $\mathrm{N} \bullet ; \mathrm{i}$ ha tenido un der.rotero propio. Pnrn ello coni111tar el libro Nt!olll1cMlúmo. U11abreve.Introduccfón. deStegery Roy.

11 Cj. Frnser. N.;El desthrn de In ig11(1/dad c11 1111 mundo Jinanciem. Conferencia dada en la Universidad Diego Po(tale\$ hups//www, youtubexom/watc.h ?v=3j8drl8 10dO. 
A su vez, la emergencia los movimientos pro modificación de las condiciones de la globalización (corno el Foro Social .iVIundiaJ), el fortalecimiento de una oplllioo pública traosnacional muchas veces apoyada por las redes sociales, la aparición del terr0túmo internacional y el terrorismo cibernético; todos estos fenómenos escán complejizando el movimien to unilineal del proyecto de consolidación de un mercado mundial globalizado.

\section{4}

Uno de los aspectos que Ja investigación de Polanyi ausculta $r$ que la antropología económica reafirma es la riqueza de los procesos de intercambios económicos, que incorporan in tercambios recíprocos de bienes e intercambios de bjeoes a través del mercado. Si conectamos este resultado con las ideas desarrolladas por Kant ensus trabajos sobre religión y sobre la virtud, podemos añadir que la sociedad no sólo realiza intercambios económicos sino también es el espacio en el cual se realizan relaciones morales bajo la forma del cumplimiento de deberes de virtud. De esta manera, es posible extraer de estos trabajos una filosofía social crítica de las relaciones sociales existen tes en la actualidad para incluir elementos normativos que permitan llevar adelante crítica y evaluación de los proyectos sociales puestos a andar en el mundo contemporáneo.

Las claves centrales de la crítica social que se extrae de esta lectura de Polanyi a la luz de la idea de comunidad ética de Kant $^{12}$ se centran especialmente en los sigujentes puntos:

a) Elproyectodelneoliberalismodedesarrai gar los mercados dela sociedad trae consigo un movimiento complemeotai-io de someter a la sociedad bajo las reglas del mercado. Ello implica la desintegración de la sociedad, de

12 La idea de comunidad éti1.:a ha sido desarrollada por Kant en su libro La religión dentro de los límites de la mero razón y puede ser valiosa para desarrollar una teoría crítica de la sociedad global contemporánea. tal manera que los intercambios recíprocos quedan marginados del análisis económico y marginados también de la práctica social. Esto acarrea el empobrecimiento al que la población es arrojada lo que reduce la capacidad de que ésta realice intercambios recíprocos como dones, de tal manera que las personas no pueden sembrar las bases del reconocimiento moral que denominamos agradecimiento.

Junto con ello, la consolidación de un mercado mundial termina por establecer relaciones de desigualdad entre regione s, como el caso de norte y sur, o entre regiones. Las relaciones económicas dentro de un mercado mundial generan relaciones de intercambio económico mercantil que somete a unos a la dominación de ocros, a la vez que los capitales transnacionalizados no sólo genera hegemonía y colonialidad en las periferias del orden mundial. Además, genera turbación en el mismo centro, pues el capital deslocalizado, busca expandir sus ganancias trasladando departamen tos empresariales enteros de un continente aJ otro, con lo cual deja en la calle a los mismos nacionales del centro del mundo globalizado, especialmente en los momentos de crisis económica como la desatada a parcir del 2008.

Al mismo tiempo, empuja a personas nacidas en las periferias a migrar, tamo de manera legal como ilega a los centros para poder acumular dinero y poder enviar remesas a los suyos, a cambio de lo cual reciben reconocimiento, prestigio $\mathrm{y}$ agradecimiento.

b) Al someter la sociedad al mercado, el proyecto neoliberal convierte el trabajo en una mercancía $y$ a las personas como engranajes del si stema. De tal manera, la dignidad de las personas tetmina siendo profundamente erosionada, pues se daña el 
tejido social en el que las relaciones sociales hacen brillar la djgni $<$ lad de las personas.

Junto a esto, la ec,momia da un vuelco inéruto en Ja historia económica de Ja humanidad: convierte al trabajo, al terreno y al runero en mercancías. El mercado de trabajo termina por convertir a la persona en una mercancía cuyo precio se encuentra regulado por las leyes del mercado en general. De esta manera, una serie de relaciones sociales se desfiguran y pierden el valor moral que la rugnidad humana les otorga.

La parcelación )' transacción económica del cerritocio se convierte en una exigencia para el mercado globalizado. Esto se debe $\mathrm{n}$ que es del territorio de donde el mercado extrae la materia prima para recrearse permanentemente. En un primer rnornemo, la parcelación del terri torio tuvo como objetivo la posibilidad de ser comprada por el capitalista agroindustrial para la siembra de algodón y el cultivo de pastizales que permitiesen ariar ganado lanero, todo ello para abastecer de materias primas a las nacientes industrias texciJes en Inglaterra duran te el siglo )...V JlJ y XIX.Ya, en el siglo XX y XXI, la expropiación del terreno a las comunidades tiene como objetivo la extracción de minerales, de petróleo $y$ de gas lo revitaliza desde las canteras formales, semiformales e infor males. Y la el cultiYo de coca, opio y marihuana lo hacen desde el lado delictivo.

La parcelación del territorio trae consigo gran conflictividad social en la que se enfrentan, de una parte, el grao capital $\mathrm{y}$, de otra, las comunidades nativas, los colonos y los ciudadanos que se ven despojados de sus territorios. Pero, a pesar de los tratados y convenios internacionales, especialmen te, el convenio 169 de la Organización Internacional del Trabajo
$(\mathrm{OlT})^{13}$, los gobiernos nacionales se colocan del lado del gran capital en cont:ra de los pobladores debido a que tales gobiernos y estados han sido copados por los agentes del capital local que busca, de esa manera, poder entablar negocios con el capital transnacional. EL fenómeno de que los gobiernos y los estados den la espalda a la sociedad para ponerse al servicio de los capitales transnacionalizados constituye una fuente profunda de cornlpción de los estados.

La conversión del runero en mercancía genera lo que se conoce como capüal especulativo, el cual se expresa en el crédiro y La especulación financiera en las bolsas locales e internacionales. La exacerbación del capital especulativo termi na por generar crisis financieros, como la del 2008, que exige que los Estados recaten a los bancos y desprotejan a las personas que tienen sus casas hipotecadas.

e) En ese mismo movimiento, el proyecto del neoliberalismo termina por imponer un proyecto de vida y la homogenización de Jos proyectos de vida, de tal manera que la persona no es considerada como un fin en sí y con la posibilidad de darse fines a sí mismos. Todo lo contrario, el mercado le impone un proyecto de vida en nombre de una idea metafisica de la realización humana general que inruca que el ser humano es u n ser racional que buscará maximizar sus ganancias y elegirá las reglas del mercado como las mejores reglas posibles. Desde esta perspectiva, Nelson Mandela, Gustavo Gutiérrez y Teresa de Calcuta serían catalogados como agentes estúpidos debido a que no actúan como agentes racionales, es decir, no procuran maximizar sus ganancias en el mercado.

13 Se puede revisar el convenio 169 de la OIT en http//www.ílo.org/ wcmsp 5/groups/public/-ed_nor m/-- aormes/docu ments/pu blication/wcms_l 00910.pdf. 


\section{UNIFÉ-EPG}

5

En el -Perú el neoliberalismo se impuso en el gobierno dictatorial de Alberto Fujimori. El conj unto de medidas económicas que dio apenas comemado su gobierno, el llamado "Fuji shock", significó en primer paso para transformar la estructura básica de la sociedad peruana. de acuerdo a las exigencias del proyecto neoliberaJ . Corno acerradameme señalan Ricardo Cuenca y los investigadores del IEP, en Jos últimos 50 años, el t.> afa tuvo dos transformaciones estr ucturales la de Velazco y la de Fujimori. La pri mera se encuen tra rep.rcentada con la Reforma Agraria y la segunda por l.a imposición dell proyecto oeoliberal en eJ Pert1. Pero cada una de estas cransfot:maciones ruvo tal profundidad que marcó desde la sociedad, pasando por la culruta y la articulación del indiviJuo en este país.

Hoy en día vivlnios bajo la sombra de la segunda tránsforma.ción. Ésra tiene. como inicio el Fujishock y modificado radicalmente la política, b sociedad y a la sabjeti.vidad en el Pen1. Esta transformación neoübetal ha uẹrado al país a una profunda descomposición social. Todas las otgani.7.aciones de la sociedad han sídc;> derretidas por eJ fuego de la trans<"tcción del dinero, y la economía se ha superpuesto sohre la politica, lo que significó la desestrucruración del sistema poiitico. Si bien, hacia fines de los 80 , el sistema poütico estaba tambaleando, 1a i:ransformación estructural que se. dio con Fujim ori tenía como 6nalidad destruirlo por complete, y Jo consiguió.

Durante los días en que se recordaron Jos 25 años la. implernentadóo de esas medidas económicas, personas Hu.nado 'Miller (gui'en fue minístro de economía de entonces y quien anunció las medidas ante las cámaras de televisión), Hernando de Saco (economista e ideólogo aeoliberal, quien escribió un famoso libro denomina.do El otro sendero (De Sot'i 1988) y miembro del grupo neoliberal denominado Instituto Libertad y Democracia), junto con otro economistas $y$ periodistas, señalaron dos aspectos que resultan ser altamente cuestionables. La primera fue que el shock era necesario, mientras ( $\mu$ e.la segunda fue gue no conespondfa a ptoyecto político alguno.

La primera afirmacíóo va en contra de un dato importanre. En ese morrtcnro, hubo un grupo de economistas y pe.csonas cercanas a Fujimori, llamados t'Los 7 srunura.is" que se opusieron al :-;hoc:k. El otro grupo lo representaban los seguidores de los "Chicago boys", ciuienes sostenían que era necesario imponer el modelo neoliberal.Hubo un debate y ganaionlos segundos) no necesariamente por9ae tuvieran mejores ideas, sino porque hubo presión política intemadonal; especialmente dd F:Nll. En ese período, era claro que había que modificar la política económica del país, peto había varios caminos posibles.

En politica, palabras como "verdad" o "necesidad ${ }^{1}$ - carecen de sentido. Nadie puede jusciftcar una medida política alegando que está inspirado pm la verdad (metafísica, religiosa o provertieme de la ciencia económica), asi como tampoco nadie puede decir que tales medidas era11 necesarias (como, por ejemplo el fupshock o las violaciones a los Derechos Humanos).

La segunda afirmación, según la cual. e) shock no correspondía a ningún proyecto polfrico, y que era u.na medida cécnic!l pa eofrentai la hJ perin.Aación, es también fulsa. Dicha medida fue el inicio de urui tra.nsformación de la estructu(3, básica de la sociedad, no sólo en su eonomfa sjno en todos sus aspectos. La clase política y sectores importantes de la sociedad ciYil opusieron resistencia a. implementación de dicho proyecto politico, lo cual condujo al gobierno a uálizar la excusa del terrorismo par.a inocular pánico en La población y a dar el golpe de estado del .5 de abril del 92. No es de e. rrañar que en orcos países de la región, l.a implemen tación del pto)recro político ncoliberal supusiera la imposidón degobiernos de facto, como en Chile y Atgeo tina.

La historia que condujo ilPerú al 11.amdo 'Fujishock" hundesuR raíces en el fin dela dictadura 
militar y el tránsito hacia la democracia. Después de 12 años en que el gobierno militar (Velazco )' Morales Bermúdez - 1968-1980) desarrolló u na forma de ejercer política a través del uso de las armas, apenas reinstaurada la democracia Sendero Luminoso reinició d desarrollo de la política armada a través de lo que denominó 'La lucha popular". La decisión de Seoc.lero Luminoso desencadenó un confücto armado interno en el Perú, en el cual se enfrentaron Sendero Luminoso y el InRTA, de uoa parte y las Fuerzas Armadas, de otra.

Jl causa de dicho confücto, la población civil se encontró atrapada entre los dos frentes, de una parce los grupos terroristas, del otro, las FE AA. En esa situación, muchas personas fueron objeto de la violación de sus derechos. Los gobiernos de Fernando Belaúnde y Alan García fueron perfeccionando sus estrategias aotisubversivas, aunque no consiguieron detener el accionar de Sendero Luminoso.

Durante elprimer gobierno de Alan García se constituyó el GEIN, que era un centro de inteligencia de la policía nacional. Este grupo consiguió dar frutos el año 92, capturando a los miembros delComité Central deSenderoLuminoso y desarticulando por completo la organización terrorista. Alberto Fujimori, Presidente de Ja República, se adjudicó el logro, pues él no esraba al tanto de las acávidades del GEIN y ni siquiera lo apoyaba. Sio embargo, Fujimori se grnnjeó una gran popularidad, lo que le permitió cerrar el Congreso e imponer una dictadura cívico -militar con lo cual logró consolidar el proyecto neolfüeral.

En ese período de 20 años, que se situó entre el 80 y el 2000, se articuló un debate que tenía tres posiciones definidas. La primera posición era consolidar la instituciooalidad democrática. La segunda posición instaurar un régimen comunista deoricntación maoístaquedestruyera lademocracia y la pluralidad de la sociedad. Finalmente, el tercer proyecto, representado por Fujimori, representó la aspirnción de instaurar una dictadura populista de derecha que impusiera el modelo neoliberal en eJ Perú. Como es sabido, este tercer proyecto fue el que venció.

Apenas iniciado el siglo X'XI la dictad ura de Fujimori se desplomó gracias a la alianza entre la colaboración internacional y el mo,cimiento de Derechos Humanos. Montesinos -el socio de Fujimori- fugó a Venezuela y el ex dictador se refugió en Japón, desde donde envió un fax aJ Congreso dela República presentando su renuncia, en un acto completamente indigno.

En ese momento se inició una transición hacia la democracia. Asimismo, se abrió un doble debate, que tenía como eje dos preguntas centrales. La primera era ¿̇ué tipo de sociedad queríamos construir?, mientras que la segunda era ¿qué modelo económjco queríamos tener? En ese momento crucial el Perú perdió una gran oportunidad, pues respondió a ambas pregun tas eligiendo las alternativas peores. A la prjmera pregunta respondió que construiría una sociedad donde el olvido respecto de los daños sufridos )' Las violaciones a los Derechos Humanos fuera el común denominador. $\mathrm{Y}$ a la segunda pregunta, respondió que seguiría aceptando el sistema económico neoliberal que la dictadura había im puesto. Es decir, oo se intentaría modificar la estructura básica de la sociedad para que la sociedad peruana sea más justa. Esta actirud se demostró con claridad el rechazo al Informe Final de la Comisión de la Verdad \}'Reconciliación.

El gobierno de tran sición de Valeotín Paniagua, que fue formado cuando el régimen dictatorial se derrumbó, constituyó una comisión de la verdad, que se denominó Comisión de la Verdad y Reconciliación (CVR) y que fue presidida por el Dr.Salomón Lemer Febres, entonces rector de la Ponáficia Uniegrsidad Católica del Perú.

La CVR tuvo como encargo realizar una investigación a fin de presentar un informe que diera cuenta de Jo sucedido durante los años del conflicto armado interno. En un plazo de tres años la CVR entregó su informe, denominado 
Informe Final de la Comisión de la Verdad $y$ Reconciliación. (IP-CVR), consciente que el relato que se presentaba era un punto de partida para iniciar un debate. Lo que el IF-CVR representaba era la constitución de una sociedad más justa e igualitaria.

Los poderes fácticos operantes eligieron bloquear la realización de ese tipo de sociedad, a fin de seguir manteniendo, eo democraci una estructura social que se encuentra gobernada por el proyecto neoliberal. Tragedjas como las de Bagua (2009) y los contantes conAictos sociales son muestra de las relaciones de inju sticia y dominación que se han instalado en la sociedad nacional.

En tales condiciones, se agudiza cada vez más la desintegración social y las personas menos favorecidas por el sistema social encuentran menos razones para cooperar con el proyecto que se ha impuesto.Esas personas ven de qué manera el proyecto neoliberal hace cada vez más precaria sus condiciones de vida $\mathrm{y}$, por lo tanto, ofrece resistencia (como en las marchas juveniles contra la llamada ciLey Pulpín'). Pero los agentes del proyecto neoliberal han articulado una ideología que se conoce como ernprendedu rismo, con el propósito de ganar el apoyo de Jas personas que se encuentran en desventaja.

La ideología deJ emprendedurismo señala el sistema económico que se ha impuesto tiene la cualidad de hacer posible que toda persona que se esfuerce un poco podrá convertirse en un empresario, y si su esfuerzo y habiJjdad es mayor, conseguirá convertirse en un empresario próspero. La ideología del emprendedurismo incorpora un discurso sobre el liderazgo según el cual, en vez de formar ciudadanos para una sociedad democrática, debemos formar emprendedores que sean lideres en una sociedad de mercado.

Ahora que el neoliberalism o es la corriente hegemónica en el mundo contemporáneo, esto trae consigo una serie de fenómenos aifadidos. Dos de ellos son la precarización de la educación y el incremento del radicalismo islámico. La precarización de la educación se encuentra representada por la escuela que se orienta a formar emprendedor es en vez de ciudadanos. Respecto de ello hemos tratado suficientemente en la primera sección del presente texto.

El radicalismo islámico es un fenómeno de la globalización que surge con el auge del neoliberalismo. En esta época de globalización, las identidades religiosas y culturales ya no saben qué lugar tienen en el mundo. Es por ello que el mundo ha devenido en un polvorín en lucha por la identidad vulnerada, tal como Amín Maalouf lo describe en su brillante ensayo Identidades asesinas. La vio.lencia que el radicalismo islámico es una de las expresiones. El radicalismo cristiano, tanto católico como elangélico, es otra de sus expresiones, ciertamente menos notoria $y$ llamativa, pero presente. El auge de la derecha cristiana, en apoyo a Donald Trump en EEUU y el auge de la derecha cristiana y católica en América Latina, en apoyo de opciones políticas autoritarias, resultan ser parte de la violencia que genern la implementación del neoliberalismo en el mundo.

Incluso, en el Mediorienrc, el radicalismo es un fenómeno de las recetas neoliberaJes instaladas. Este efecto deja un saldo extremadamente negativo para la formación de las personas a Jo largo y ancho del mu ndo. En vez de mirar aJ otro con soljdaridad, curiosidad y aprecio, las persona s están siendo formadas para mirar al otro con desprecio, desconfianza y una profunda ignorancia. Lo peor de todo, es que la educación se está enrumbando a formar a seres humanos desconfiados $\mathrm{v}$ temerosos de los demás, arrojando un saldo completamente negativo para lo que a solidaridad se refiere.

\section{BIBLIOGRAFiA}

De Soto, H. (1988) El otro .re11dero, Lima: El Barranco. 
rrnser, N. (2998) Escalas de j11sti cia, Barcelona: Herder.

Hobbes, T. (2004) Leviatán: o la motena,far111 a J poder de 1111 e.rtado et lesiásticoy civil Madrid: Alianza.

llonneth,A. (2009) Critica del agmvio/J/Ora/.Patologías de la sociedad co11temporcíma, México: FCE.

Mauss, M. (2009) E11sf!YO sobre t:! do11:foona)'f1111ció11 del i11terca111bio en la.s sociedades arcaicas, Bs. ils.: Katz.

Negri, A. (2003) CJisú de la político: esetitos sobre J1arx, K'!)1m.r, las crisis corpita!listc1sy las 1111evas mqjetividadu, Bs. As.: El cielo por asalto.

Nusbaum, M. (2010) Sin Ji11 U ele lucro:por qlféla de111omlaia necesita de las IJ11111a11idades, 1 \fadrid:

Katz.

Polanyi, K. (2010) La gran /m11sjor111ación, México: FCE.

Posner, R. (2011) Análisis 11co11ó111iro del derecho, Bogota: Siglo del Hombre: Universidad de los Andes: Pontificia Universidad Javeriana. Instituto Pensar.

Sahlins, M. (1983) Ec01tomío de IP edad de piedra, Madrid: Akal.

Weber, M.(2001)La ética protestante)'el 'e.spiri/11"del capitolis1110, Madrid: Alianza. 